\title{
The New Approach to Investigation of Multilayer Graphene Mechanical Properties by the Finite-element Method
}

\section{O. E. Glukhova, A. V. Dol', A. S. Kolesnikova, V. V. Shunaev}

Saratov State University, 83, Astrahanskaya str., 410012, Saratov, Russia, oeglukhova@yandex.ru, dzero@pisem.net, kolesnikova.88@mail.ru, vshunaev@list.ru

A new approach to investigate the mechanical properties of multilayer graphene was suggested. The method is based on the idea that the van der Waals interaction between the graphene sheets can be simulated by a fictitious layer of continuum. The stress-strain state of multilayer graphene is described by stationary equations of Navier-Lame. This approach has been successfully tested on graphene deflection. The graphene layers were considered as linear-elastic material. For each part of the curve that approximates the dependence of the graphene deflection on the applied force, corresponding elastic constants of graphene layers were found.

Key words: multilayer graphene, finite element method, deflection, modulus of elasticity, van der Waals interactions

\section{References}

1. Gil A. J., Adhikari S., Scarpa F., Bonet J. The formation of wrinkles in single-layer graphene sheets under nanoindentation. J. Phys. Condens. Matter., 2010, vol. 22, no. 14 , pp. 145302-1-145302-6. DOI: 10.1088/0953$8984 / 22 / 14 / 145302$

2. Wang Z., Laetitia P., Jamil E. Deflection of suspended graphene by a transverse electric field. Physical Review B., 2009, vol. 81, iss. 15, pp. 155405-1-155405-5.

3. Glukhova O. E., Shunaev V. V. Investigation of the tensile strength of mono-and bilayer graphene J. Nano and microsystem technique, 2012, no. 7, pp. 25-29 (in Russian)
4. Lee C., Wei X., Li Q., Carpick R., Kysar J. W., Hone J. Elastic and frictional properties of graphene. Physica Status Solidi, 2009, vol. 246, no. 11-12, pp. 2562-2567. 5. Rouhi S., Ansari R. Atomistic finite element model for axial buckling and vibration analysis of single-layered graphene sheets. Physica E : Low-dimensional Systems and Nanostructures, 2012, vol. 44, iss. 4, pp. 764-772. 6. Mikhailov S. Physics and Applications of GrapheneTheory. Rijeka, Croatia, InTech, 2011, 534 p.

7.Nahas M. N., Abd-Rabou M. Finite element modeling of carbon nanotubes. Intern. J. of Mechanical and Mechatronics IJMME-IJENS, 2010, vol. 10, no. 3, pp. 19-24.

УДК 539.374

\section{МАТЕМАТИЧЕСКАЯ ТЕОРИЯ СВЯЗАННЫХ ПЛОСКИХ ГАРМОНИЧЕСКИХ ТЕРМОУПРУГИХ ВОЛН В МИКРОПОЛЯРНЫХ КОНТИНУУМАХ ПЕРВОГО ТИПА}

\author{
В. А. Ковалев ${ }^{1}$, Е. В. Мурашкин ${ }^{2}$, Ю. Н. Радаев ${ }^{3}$
}

\begin{abstract}
${ }^{1}$ Доктор фризико-математических наук, просрессор касредры управления проектами и инвестициями, Московский городской университет управления Правительства Москвы, vlad_koval@mail.ru

${ }^{2}$ Кандидат фризико-математических наук, научный сотрудник, Институт проблем механики им. А. Ю. Ишлинского РАН, Mоckвa, murashkin@ipmnet.ru, evmurashkin@gmail.com

${ }^{3}$ Доктор фризико-математических наук, просрессор, ведущий научный сотрудник, Институт проблем механики им. А. Ю. Ишлинского РАН, Москва, radayev@ipmnet.ru, y.radayev@gmail.com
\end{abstract}

\begin{abstract}
В представляемой работе в рамках линейной теории обобщенной микрополярной термоупругости первого типа (GNI/CTE) с помощью связанной системы уравнений движения и теплопроводности выполнен анализ плоских гармонических связанных термоупругих волн перемещений, микровращений и температуры. Исследованы также закономерности распространения волновых поверхностей слабых разрывов перемещений, микровращений и температуры в термоупругом микрополярном континууме первого типа. Вычислены нормальные скорости распространения указанных волновых поверхностей. Получено и проанализировано с помощью пакета символьных вычислений Mathematica детерминантное уравнение для определения волновых чисел (постоянных распространения (РС)) плоских гармонических связанных термоупругих волн перемещений,микровращений и температуры. Факторизация полученного частотного полиномиального уравнения 14-й степени позволила свести его к биквадратному и бикубическому уравнениям относительно волновых чисел. Для волновых чисел поперечных и продольных волн получены алгебраические формы, содержащие многозначные комплексные квадратные и кубические радикалы.
\end{abstract}

Ключевые слова: микрополярная термоупругость, континуум первого типа, слабый разрыв, продольная волна, поперечная волна, волновое число, частотное уравнение. 


\section{ВВЕДЕНИЕ}

Проблемы деформации континуумов, когда наряду с обычными перемещениями и возможны также микровращения $\phi$, постоянно привлекают внимание исследователей, начиная с классической работы Э. Коссера и Ф. Коссера (E. et F. Cosserat) [1]. В подобных моделях, в отличие от классических теорий, состояние континуума описывается несимметричными тензорами деформаций и напряжений, поэтому упругие тела в рамках несимметричной теории характеризуются достаточно большим числом определяющих упругих постоянных. Необходимость подобного усложнения объясняется невозможностью в рамках классической теории упругости (и пьезоэлектроупругости) объяснить, например, аномальный пьезоэффект в кварце, дисперсию упругих волн, а также ряд других экспериментально наблюдаемых упругих свойств чистых кристаллов.

В настоящее время быстро развиваются математические модели термоупругого поведения твердых тел (GN-термоупругость), основанные на различных модификациях закона теплопроводности Фурье. При этом ставится цель получения связанных гиперболических уравнений термоупругости, которые гарантировали бы выполнение следующих условий: 1) конечность скорости распространения теплового сигнала; 2) возможность пространственного распространения теплового импульса без затухания; 3) отсутствие искажения волны в смысле получения решений классического даламберовского типа с сохраняющимся профилем. Одно из таких направлений связано с публикациями [2, 3]. GN-теории термоупругости можно разделить на три различных варианта: термоупругость, основанную на законе теплопроводности Фурье, с бесконечно большой скоростью распространения экспоненциально затухающего теплового сигнала (континуум первого типа, GNI/CTE); термоупругость с сохраняющейся энергией и конечной скоростью распространения термических волн «второго звука» (континуум второго типа, GNII, гиперболическая термоупругость); третий вариант (континуум третьего типа, GNIII) смешанный и включает первые два в качестве предельных случаев. Очевидно, что GNIIIтеория охватывает более широкий круг моделируемых явлений по сравнению с классической теорией теплопроводности Фурье. Отметим, что GNII - единственная известная в настоящее время термодинамически корректная теория, которая позволяет описать теплопроводность в твердом теле как волновой процесс, не сопровождающийся рассеянием энергии, и сформулировать математическую модель процесса теплопроводности в твердых телах с помощью системы гиперболических уравнений в частных производных, обеспечивающих (в силу своей аналитической классификации) конечную скорость распространения тепла.

Полный анализ плоских гармонических GNI/CTE-термоупругих волн был выполнен в статье [4], было показано, что для каждой частоты всегда имеется ровно два комплексных волновых числа, вещественная и мнимая части которых строго положительны. Плоские волны в GNII-термоупругих средах исследованы в работе [5]. Плоские термоупругие волны «второго звука» характеризуются четырьмя вещественными волновыми числами, два из которых положительны. В работе [6] проведен анализ волновых чисел плоских гармонических связанных термоупругих волн, которые описываются линейными уравнениями GNIII-термоупругости. Все указанные исследования [4-6] проводились в рамках линейной симметричной упругости. Они подытожены в монографии [7].

В настоящем исследовании ставится задача вычисления волновых чисел плоских гармонических термоупругих волн в GNI/CTE-термоупругих микрополярных средах при наличии микровращений. Исследованы также закономерности распространения поверхностей слабых разрывов перемещений, микровращений и температуры в термоупругом микрополярном континууме первого типа. Вычислены скорости распространения поверхностей слабых разрывов. Получено и проанализировано с помощью пакета символьных вычислений Mathematica детерминантное уравнение для определения волновых чисел плоских гармонических связанных термоупругих волн перемещений, микровращений и температуры. Факторизация полученного частотного полиномиального уравнения 14-й степени позволила свести его к биквадратному и бикубическому уравнениям относительно волновых чисел, что затем без труда позволяет найти волновые числа плоских гармонических связанных термоупругих поперечных и продольных волн. 


\section{1. ОСНОВНЫЕ УРАВНЕНИЯ СВЯЗАННОЙ МИКРОПОЛЯРНОЙ ТЕРМОУПРУГОСТИ ПЕРВОГО ТИПА (GNI/СТЕ)}

Воспользуемся моделью термоупругого микрополярного континуума первого типа (см., например, $[8,9])$. В этом случае векторы перемещений $\mathbf{u}$ и микровращений $\phi$ связаны с несимметричным тензором деформации $\boldsymbol{e}$ и тензором изгиба-кручения $\boldsymbol{\Gamma}$ соотношениями

$$
\begin{gathered}
e=\nabla \otimes \mathbf{u}-\epsilon \cdot \phi, \\
\Gamma=\nabla \otimes \phi,
\end{gathered}
$$

где $\boldsymbol{\epsilon}-$ кососимметричный тензор Леви-Чивита третьего ранга, $\boldsymbol{\nabla}-$ трехмерный оператор Гамильтона (набла Гамильтона).

Несимметричный тензор силовых напряжений $\boldsymbol{\sigma}$ и тензор моментных напряжений $\boldsymbol{m}$ вычисляются согласно определяющему закону GNI/CTE-термоупругости:

$$
\begin{gathered}
\boldsymbol{\sigma}=(\mu+\eta) \boldsymbol{e}+(\mu-\eta) \boldsymbol{e}^{\mathrm{T}}+(\lambda \operatorname{tr} \boldsymbol{e}-\alpha \theta) \mathbf{I} \\
\boldsymbol{m}=(\gamma+\varepsilon) \boldsymbol{\Gamma}+(\gamma-\varepsilon) \boldsymbol{\Gamma}^{\mathrm{T}}+(\beta \operatorname{tr} \boldsymbol{\Gamma}-\varsigma \theta) \mathbf{I}
\end{gathered}
$$

Здесь I - единичный тензор; $\lambda, \mu, \eta, \gamma, \beta, \varepsilon$ - изотермические определяющие постоянные микрополярной термоупругой среды первого типа; $\alpha, \varsigma$ - определяющие постоянные, обеспечивающие связанность уравнений движения и теплопроводности. Постоянные $\alpha$, ऽ зависят не только от механических свойств среды, но и от термических свойств.

Уравнения движения микрополярной среды запишем в предположении отсутствия массовых сил и массовых моментов:

$$
\begin{gathered}
\nabla \cdot \boldsymbol{\sigma}=\rho \ddot{\mathbf{u}}, \\
\boldsymbol{\epsilon} \cdot \boldsymbol{\sigma}+\boldsymbol{\nabla} \cdot \boldsymbol{m}=\mathfrak{J} \ddot{\phi} .
\end{gathered}
$$

где $\rho$ - плотность среды, $\mathfrak{J}$ - мера микроинерции среды при вращении, точка над символом обозначает частное дифференцирование по времени при фиксированных пространственных координатах.

Уравнения движения (3) следует дополнить уравнением теплопроводности. Его нетрудно получить, воспользовавшись законом распространения тепла Фурье, который устанавливает пропорциональность вектора потока тепла $\mathbf{h}$ и отрицательного градиента температуры $\theta$ :

$$
\mathbf{h}=-\Lambda_{*} \nabla \theta,
$$

где $\Lambda_{*}-$ коэффициент теплопроводности, $\Lambda_{*}>0$.

Если подставить напряжения $\boldsymbol{\sigma}$ и $\boldsymbol{m}$ из формул (2) в уравнения движения (3) и выразить тензоры $\boldsymbol{\Gamma}$ и $\boldsymbol{e}$ через перемещения $\mathbf{u}$ и микровращения $\phi$, то получим замкнутую систему связанных уравнений движения и теплопроводности для линейного изотропного микрополярного термоупругого тела первого типа при условии отсутствия массовых сил, моментов и источников тепла $[8,10]$ :

$$
\left\{\begin{array}{l}
(\lambda+\mu-\eta) \boldsymbol{\nabla} \nabla \cdot \mathbf{u}+(\mu+\eta) \boldsymbol{\nabla}^{2} \mathbf{u}+2 \eta \boldsymbol{\nabla} \times \boldsymbol{\phi}-\alpha \boldsymbol{\nabla} \theta-\rho \ddot{\mathbf{u}}=\mathbf{0}, \\
(\beta+\gamma-\varepsilon) \boldsymbol{\nabla} \boldsymbol{\nabla} \cdot \boldsymbol{\phi}+(\gamma+\varepsilon) \boldsymbol{\nabla}^{2} \boldsymbol{\phi}-4 \eta \boldsymbol{\phi}+2 \eta \boldsymbol{\nabla} \times \mathbf{u}-\varsigma \boldsymbol{\nabla} \theta-\mathfrak{J} \ddot{\boldsymbol{\phi}}=\mathbf{0}, \\
\boldsymbol{\nabla}^{2} \theta-\frac{\kappa}{\Lambda_{*}} \dot{\theta}-\frac{\alpha}{\Lambda_{*}} \boldsymbol{\nabla} \cdot \dot{\mathbf{u}}-\frac{\varsigma}{\Lambda_{*}} \boldsymbol{\nabla} \cdot \dot{\boldsymbol{\phi}}=0 .
\end{array}\right.
$$

Здесь $\boldsymbol{\nabla}^{2}=\boldsymbol{\nabla} \cdot \boldsymbol{\nabla}$ - трехмерный оператор Лапласа, $\theta$ - приращение температуры над отсчетной температурой, $\theta_{0}$ - отсчетная температура, $\Lambda_{*}-$ коэффициент теплопроводности (коэффициент термической диффузии), $\kappa$ - теплоемкость (на единицу объема) при постоянной деформации.

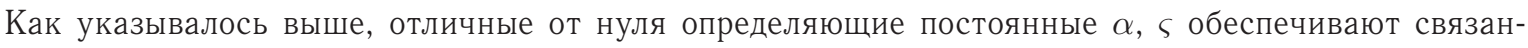
ность уравнений микрополярной термоупругости. Обычно считается, что $\varsigma=0$ [10]. Ради полноты анализа мы сохраним эту определяющую постоянную во всех дальнейших рассуждениях.

Скалярное уравнение в системе (4) называется обобщенным уравнением теплопроводности, сопряженным с уравнениями движения (первое и второе уравнения в системе (4)). 


\section{2. СЛАБЫЕ РАЗРЫВЫ ТЕРМОМЕХАНИЧЕСКИХ ПОЛЕЙ В ТЕРМОУПРУГОМ МИКРОПОЛЯРНОМ КОНТИНУУМЕ ПЕРВОГО ТИПА}

Исследуем сначала закономерности распространения слабых разрывов перемещений $\mathbf{u}$, микровращений $\phi$ и температуры $\theta$ в термоупругом микрополярном континууме первого типа. Отметим, что система дифференциальных уравнений в частных производных (4) содержит частные производные не выше второго порядка. Пусть в трехмерном пространстве с нормальной скоростью $G$ распространяется фронт (волновая поверхность) слабых разрывов перемещений $\mathbf{u}$, микровращений $\boldsymbol{\phi}$ и температуры $\theta$. Обозначим через $\mathbf{n}$ единичный вектор нормали к указанной волновой поверхности. Тогда геометрические и кинематические условия совместности второго порядка Адамара-Томаса будут иметь вид ${ }^{1}$

$$
\begin{array}{ll}
{[\boldsymbol{\nabla} \otimes \boldsymbol{\nabla} \otimes \mathbf{u}]=\mathbf{n} \otimes \mathbf{n} \otimes \mathbf{A},} & {[\boldsymbol{\nabla} \otimes \boldsymbol{\nabla} \otimes \boldsymbol{\phi}]=\mathbf{n} \otimes \mathbf{n} \otimes \mathbf{S},} \\
{[\boldsymbol{\nabla} \otimes \dot{\mathbf{u}}]=-G \mathbf{n} \otimes \mathbf{A},} & {[\boldsymbol{\nabla} \otimes \dot{\boldsymbol{\phi}}]=-G \mathbf{n} \otimes \mathbf{S},} \\
{[\ddot{\mathbf{u}}]=G^{2} \mathbf{A},} & {[\ddot{\boldsymbol{\phi}}]=G^{2} \mathbf{S},} \\
{[\boldsymbol{\nabla} \otimes \boldsymbol{\nabla} \theta]=B \mathbf{n} \otimes \mathbf{n},} &
\end{array}
$$

где квадратные скобки обозначают скачок при переходе через поверхность слабого разрыва. $B, \mathbf{A}, \mathbf{S}-$ некоторые поля, определенные на этой поверхности, причем равенства $B=0, \mathbf{A}=\mathbf{0}, \mathbf{S}=\mathbf{0}$ не могут выполняться одновременно ни в какой точке поверхности, если рассматриваемая поверхность действительно является поверхностью слабого разрыва. Уравнения (4) вместе с (5) дают следующие соотношения, связывающие скачки частных производных второго порядка от перемещений $\mathbf{u}$, микровращений $\phi$ и температуры $\theta$ при переходе через волновую поверхность:

$$
\left\{\begin{array}{l}
\left(\rho G^{2}-(\mu+\eta)\right) \mathbf{A}-(\lambda+\mu-\eta) \mathbf{n}(\mathbf{n} \cdot \mathbf{A})=\mathbf{0}, \\
\left(\mathfrak{J} G^{2}-(\gamma+\varepsilon)\right) \mathbf{S}-(\beta+\gamma-\varepsilon) \mathbf{n}(\mathbf{n} \cdot \mathbf{S})=\mathbf{0}, \\
B-\frac{\alpha G}{\Lambda_{*}} \mathbf{n} \cdot \mathbf{A}-\frac{\varsigma G}{\Lambda_{*}} \mathbf{n} \cdot \mathbf{S}=0 .
\end{array}\right.
$$

Вычислим далее проекции системы (6) на нормальное $\mathbf{n}$ и касательное $\boldsymbol{\tau}$ направления к волновой поверхности. При проектировании системы уравнений (6) третье скалярное уравнение не измененяется. Проекция векторных уравнений (6) на нормальное направление $\mathbf{n}$ приводит к следующей однородной системе уравнений:

$$
\left\{\begin{array}{l}
\left(\rho G^{2}-(\lambda+2 \mu)\right)(\mathbf{n} \cdot \mathbf{A})=0, \\
\left(\mathfrak{J} G^{2}-(\beta+2 \gamma)\right)(\mathbf{n} \cdot \mathbf{S})=0, \\
B-\frac{\alpha G}{\Lambda_{*}} \mathbf{n} \cdot \mathbf{A}-\frac{\varsigma G}{\Lambda_{*}} \mathbf{n} \cdot \mathbf{S}=0 .
\end{array}\right.
$$

Если $\mathbf{n} \cdot \mathbf{A}=0, \mathbf{n} \cdot \mathbf{S}=0$, тогда $B=0$ и, возвращаясь к (6), получаем, что скалярное уравнение в (6) удовлетворяется тождественно; если оба множителя при векторах поляризации отличны от нуля, то $\mathbf{A}=\mathbf{0}, \mathbf{S}=\mathbf{0}$, и поверхность не является поверхностью слабого разрыва.

В итоге остается три различных случая:

І случай $-\mathbf{n} \cdot \mathbf{A} \neq 0, \mathbf{n} \cdot \mathbf{S}=0$;

II случай $-\mathbf{n} \cdot \mathbf{A}=0, \mathbf{n} \cdot \mathbf{S} \neq 0$;

III случай $-\mathbf{n} \cdot \mathbf{A} \neq 0, \mathbf{n} \cdot \mathbf{S} \neq 0$.

Рассмотрим последовательно каждый из перечисленных выше случаев. В первом случае выполнение первого уравнения системы (7) возможно только на волновой поверхности, распространяющейся с нормальной скоростью:

$$
G=c_{\|},
$$

где $c_{\|}=\sqrt{(\lambda+2 \mu) / \rho}$.

\footnotetext{
${ }^{1}$ Соответствующая теория геометрических и кинематических условий совместности изложена, например, в известной монографии,см.: Томас T. Пластическое течение и разрушение в твердых телах. М.: Мир, 1964. 308 с.
} 
В этом случае нормальная скорость распространения поверхности слабого разрыва $G(8)$ оказывается равной скорости чисто упругих продольных волн $c_{\|}$. Кроме того, согласно третьему уравнению системы (7) при переходе через указанную волновую поверхность поле температур также претерпевает слабый разрыв:

$$
B=\frac{\alpha G}{\Lambda_{*}} \mathbf{n} \cdot \mathbf{A} .
$$

Второй случай подразумевает наличие слабого разрыва микровращений $\mathbf{n} \cdot \mathbf{S} \neq 0$. Тогда выполнение второго уравнения системы (7) возможно только на поверхности слабого разрыва, распространяющейся с нормальной скоростью:

$$
G=c_{\|}^{\mu \mu}
$$

где $c_{\|}^{\mu \mu}=\sqrt{(\beta+2 \gamma) / \mathfrak{J}}$ есть скорость волны кручения. Как и в предыдущем случае, указанный слабый разрыв микровращений связан со слабым разрывом поля температур третьим уравнением системы (7):

$$
B=\frac{\varsigma G}{\Lambda_{*}} \mathbf{n} \cdot \mathbf{S}
$$

Таким образом, определяющие постоянные $\alpha, \varsigma$ характеризуют интенсивность слабого температурного разрыва.

В третьем случае множители при $\mathbf{n} \cdot \mathbf{A}$ и $\mathbf{n} \cdot \mathbf{S}$ должны одновременно обращаться в нуль, что невозможно, если $c_{\|} \neq c_{\|}^{\mu \mu}$. Ясно, что при этом рассматриваемая поверхность не будет волновой. Если континуум можно охарактеризовать определяющим равенством $c_{\|}=c_{\|}^{\mu \mu}$, то волновая поверхность будет волной, распространяющейся со скоростью

$$
G=c_{\|}=c_{\|}^{\mu \mu}
$$

а слабый разрыв поля температуры находится из условия

$$
B=\frac{\alpha G}{\Lambda_{*}} \mathbf{n} \cdot \mathbf{A}+\frac{\varsigma G}{\Lambda_{*}} \mathbf{n} \cdot \mathbf{S} .
$$

Соотношение (10) подразумевает возможность бездиссипативного распространения продольной волны при выполнении условия $B=0$, т. е. когда

$$
\frac{\alpha}{\varsigma}=-\frac{\mathbf{n} \cdot \mathbf{A}}{\mathbf{n} \cdot \mathbf{S}}
$$

Вычислим проекцию системы уравнений (6) на произвольное касательное направление $\tau$ к волновой поверхности, третье скалярное уравнение переписывается без изменений:

$$
\left\{\begin{array}{l}
\left(\rho G^{2}-(\mu+\eta)\right)(\boldsymbol{\tau} \cdot \mathbf{A})=0, \\
\left(\mathfrak{J} G^{2}-(\gamma+\varepsilon)\right)(\boldsymbol{\tau} \cdot \mathbf{S})=0, \\
B-\frac{\alpha G}{\Lambda_{*}} \mathbf{n} \cdot \mathbf{A}-\frac{\varsigma G}{\Lambda_{*}} \mathbf{n} \cdot \mathbf{S}=0 .
\end{array}\right.
$$

Вновь возможны три различных случая:

І случай $-\boldsymbol{\tau} \cdot \mathbf{A} \neq 0, \boldsymbol{\tau} \cdot \mathbf{S}=0$;

II случай $-\boldsymbol{\tau} \cdot \mathbf{A}=0, \boldsymbol{\tau} \cdot \mathbf{S} \neq 0$;

III случай $-\boldsymbol{\tau} \cdot \mathbf{A} \neq 0, \boldsymbol{\tau} \cdot \mathbf{S} \neq 0$.

Первый случай подразумевает наличие слабого разрыва перемещений, который возможен лишь на поверхности, распространяющейся со скоростью

$$
G=c_{\perp}^{\mu},
$$

где $c_{\perp}^{\mu}=\sqrt{(\mu+\eta) / \rho}$.

Заметим, что при равенстве нулю определяющей микрополярной постоянной $\eta$ скорость $G(12)$ в точности совпадет со скоростью чисто упругой поперечной волны $c_{\perp}=\sqrt{\mu / \rho}$. 
Во втором случае второе уравнение системы (11) позволяет определить скорость распространения слабого разрыва микровращений:

$$
G=c_{\perp}^{\mu \mu}
$$

где

$$
c_{\perp}^{\mu \mu}=\sqrt{\frac{\gamma+\varepsilon}{\mathfrak{J}}} .
$$

Заметим, что слабый разрыв температуры никак не связан с касательными составляющими слабого разрыва полей $\mathbf{u}, \boldsymbol{\phi}$.

В третьем случае множители при $\boldsymbol{\tau} \cdot \mathbf{A}$ и $\boldsymbol{\tau} \cdot \mathbf{S}$ не могут одновременно обращаться в нуль, если $c_{\perp}^{\mu} \neq c_{\perp}^{\mu \mu}$. Если континуум допускает совпадение скоростей поперечных волн $c_{\perp}^{\mu}=c_{\perp}^{\mu \mu}$, то скорость распространения волновой поверхности оказывается равной

$$
G=c_{\perp}^{\mu}=c_{\perp}^{\mu \mu}
$$

Слабый разрыв поля температуры вновь определяется из условия (10) и он исчезает при отсутствии нормальных проекций векторов поляризации.

Отметим, что в случаях (12), (13) поверхности слабых разрывов в термоупругой микрополярной среде типа GNI/CTE могут распространяться в отсутствие слабых разрывов температурного поля, что означает бездиссипативный механизм распространения подобных поверхностей.

\section{3. ПЛОСКИЕ ГАРМОНИЧЕСКИЕ СВЯЗАННЫЕ ТЕРМОУПРУГИЕ ВОЛНЫ В МИКРОПОЛЯРНОМ КОНТИНУУМЕ ПЕРВОГО ТИПА}

Плоская гармоническая связанная микрополярная термоупругая волна обладает достаточно простой аналитической структурой вида

$$
\mathbf{u}=\mathbf{A} e^{i(\mathbf{k} \cdot \mathbf{r}-\omega t)}, \quad \phi=\mathbf{S} e^{i(\mathbf{k} \cdot \mathbf{r}-\omega t)}, \quad \theta=B e^{i(\mathbf{k} \cdot \mathbf{r}-\omega t)},
$$

где $\mathbf{k}-$ волновой вектор, $\omega-$ циклическая частота; $\mathbf{A}, \mathbf{S}-$ векторы поляризации волны; $B-$ амплитуда отклонений температуры от отсчетной температуры. Не следует путать с обозначениями п. 2, где $\mathbf{A}, \mathbf{S}, B$ - величины слабых разрывов полей перемещений, микровращений и температуры соответственно. Волновое число $k$ (модуль волнового вектора $\mathbf{k}$ или постоянная распространения волны) может быть как вещественной величиной, так и комплексной.

Заметим, что в физической литературе волновым числом обычно называется $\operatorname{Re} k$; величина $\operatorname{Im} k$ называется коэффициентом затухания. С волновым числом $k$ связана также следующая терминология:

$$
\begin{array}{ll}
\text { фазовая скорость волны } & -\frac{\omega}{\operatorname{Re} k}, \\
\text { глубина проникания волны } & -\frac{1}{\operatorname{Im} k}, \\
\text { коэффициент потерь } & -4 \pi\left|\frac{\operatorname{Im} k}{\operatorname{Re} k}\right| .
\end{array}
$$

Подставив выражения (14) в дифференциальные уравнения (4), а также учитывая, что

$$
\boldsymbol{\nabla}=i \mathbf{k}, \quad \partial / \partial t=-i \omega
$$

получим приведенную ниже систему уравнений, связывающую волновой вектор $\mathbf{k}$, циклическую частоту $\omega$, векторы поляризации плоской волны $\mathbf{A}, \mathbf{S}$ и амплитуду $B$ :

$$
\left\{\begin{array}{l}
\left(\rho \omega^{2}-(\mu+\eta) k^{2}\right) \mathbf{A}-(\lambda+\mu-\eta) \mathbf{k}(\mathbf{k} \cdot \mathbf{A})+2 \eta i \mathbf{k} \times \mathbf{S}-\alpha i \mathbf{k} B=\mathbf{0}, \\
\left(\mathfrak{J} \omega^{2}-4 \eta-(\gamma+\varepsilon) k^{2}\right) \mathbf{S}-(\beta+\gamma-\varepsilon) \mathbf{k}(\mathbf{k} \cdot \mathbf{S})+2 \eta i \mathbf{k} \times \mathbf{A}-\varsigma i \mathbf{k} B=\mathbf{0}, \\
\left(\frac{\kappa}{\Lambda_{*}} i \omega-k^{2}\right) B-\frac{\alpha}{\Lambda_{*}} \omega \mathbf{k} \cdot \mathbf{A}-\frac{\varsigma}{\Lambda_{*}} \omega \mathbf{k} \cdot \mathbf{S}=0 .
\end{array}\right.
$$


Полученную систему линейных уравнений перепишем как следующую формальную линейную алгебраическую систему уравнений относительно комплексных амплитуд:

$$
\mathrm{D} \cdot \mathfrak{a}=\mathbf{0},
$$

где вводится формальный вектор-столбец $\mathfrak{a}=(\mathbf{A}, \mathbf{S}, B)^{\mathrm{T}}$.

В том случае, когда волновой вектор $\mathbf{k}$ сонаправлен с одной из координатных осей (например, с декартовой осью $x_{3}$ ), а этого всегда можно добиться за счет поворота координатных осей, комплексная матрица $\mathbf{D}$ будет обладать достаточно простой структурой. Несложные вычисления показывают, что матрица D будет иметь следующий вид:

$$
\left(\begin{array}{ccccccc}
d_{11} & 0 & 0 & 0 & d_{15} & 0 & 0 \\
0 & d_{22} & 0 & d_{24} & 0 & 0 & 0 \\
0 & 0 & d_{33} & 0 & 0 & 0 & d_{37} \\
0 & d_{42} & 0 & d_{44} & 0 & 0 & 0 \\
d_{51} & 0 & 0 & 0 & d_{55} & 0 & 0 \\
0 & 0 & 0 & 0 & 0 & d_{66} & d_{67} \\
0 & 0 & d_{73} & 0 & 0 & d_{76} & d_{77}
\end{array}\right) .
$$

Не будем пока останавливаться на конкретизации элементов $d_{i j}$ матрицы D. Преобразуем ее, прежде всего, к блочному виду. Если переставить местами 3-ю и 5-ю строки матрицы $\mathbf{D}$ и 3-й и 5-й столбцы, а затем 2-ю и 3-ю строки и 2-й и 3-й столбцы, то матрица приводится к блочно-диагональной форме:

$$
\left(\begin{array}{ccc}
\mathbf{D}_{1} & \mathbf{0} & \mathbf{0} \\
\mathbf{0} & \mathbf{D}_{2} & \mathbf{0} \\
\mathbf{0} & \mathbf{0} & \mathbf{D}_{3}
\end{array}\right)
$$

Здесь составляющие блоки $\mathbf{D}_{1}, \mathbf{D}_{2}, \mathbf{D}_{3}$ представляются через элементы $d_{i j}$ исходной матрицы $\mathbf{D}$ следующими соотношениями:

$$
\begin{gathered}
\mathbf{D}_{1}=\left(\begin{array}{ll}
d_{11} & d_{15} \\
d_{51} & d_{55}
\end{array}\right)=\left(\begin{array}{cc}
c_{\perp}^{\mu 2} k^{2}-\omega^{2} & \frac{2 i \eta k}{\rho} \\
-\frac{2 i \eta k}{\mathfrak{J}} & c_{\perp}^{\mu \mu 2} k^{2}-\omega^{2}+\frac{4 \eta}{\mathfrak{J}}
\end{array}\right), \\
\mathbf{D}_{2}=\left(\begin{array}{ll}
d_{22} & d_{24} \\
d_{42} & d_{44}
\end{array}\right)=\left(\begin{array}{cc}
c_{\perp}^{\mu 2} k^{2}-\omega^{2} & -\frac{2 i \eta k}{\rho} \\
\frac{2 i \eta k}{\mathfrak{J}} & c_{\perp}^{\mu \mu 2} k^{2}-\omega^{2}+\frac{4 \eta}{\mathfrak{J}}
\end{array}\right), \\
\mathbf{D}_{3}=\left(\begin{array}{ccc}
d_{33} & 0 & d_{37} \\
0 & d_{66} & d_{15} \\
d_{73} & d_{76} & d_{77}
\end{array}\right)=\left(\begin{array}{ccc}
c_{\|}^{2} k^{2}-\omega^{2} & 0 & \frac{i \alpha k}{\rho} \\
0 & c_{\|}^{\mu \mu 2} k^{2}-\omega^{2}+\frac{4 \eta}{\mathfrak{J}} & \frac{i \varsigma k}{\mathfrak{J}} \\
\frac{\alpha \omega k}{\Lambda_{*}} & \frac{\varsigma \omega k}{\Lambda_{*}} & k^{2}-\frac{i \omega}{l_{*}^{2}}
\end{array}\right) .
\end{gathered}
$$

Для нетривиальной разрешимости системы (16) необходимо выполнение условия равенства нулю определителя матрицы D. Учитывая представление (17), определитель вычисляется проще. При этом, очевидно, выполняется равенство

$$
\operatorname{det} \mathbf{D}_{1}=\operatorname{det} \mathbf{D}_{2}
$$

В результате определитель матрицы $\mathbf{D}$ получается как

$$
\operatorname{det} \mathbf{D}=\left(\operatorname{det} \mathbf{D}_{1}\right)^{2} \operatorname{det} \mathbf{D}_{3}=\left(d_{11} d_{55}-d_{15} d_{51}\right)^{2}\left(d_{33} d_{66} d_{77}-d_{33} d_{67} d_{76}-d_{66} d_{37} d_{73}\right) .
$$

Вычисления были проверены в системе символьных вычислений Mathematica. Процедура факторизации дает тот же результат (18). Условие $\operatorname{det} \mathbf{D}=0$ будет выполнено, если хотя бы один из 
сомножителей в формуле (18) будет равен нулю, что приводит к биквадратному $\operatorname{det} \mathbf{D}_{1}=0$ и бикубическому $\operatorname{det} \mathbf{D}_{3}=0$ уравнениям относительно неизвестного волнового числа $k$. Причем из биквадратного уравнения определяются скорости распространения поперечных волн, а из бикубического скорости продольных волн.

\section{4. ВОЛНОВЫЕ ЧИСЛА ПЛОСКИХ ГАРМОНИЧЕСКИХ ПОПЕРЕЧНЫХ ВОЛН В КОНТИНУУМАХ ПЕРВОГО ТИПА}

Исследование распространения плоских гармонических поперечных волн начнем с нахождения корней (волновых чисел) биквадратного уравнения:

$$
\operatorname{det} \mathbf{D}_{1}=d_{11} d_{55}-d_{15} d_{51}=\left(c_{\perp}^{\mu 2} k^{2}-\omega^{2}\right)\left(c_{\perp}^{\mu \mu 2} k^{2}-\omega^{2}+\Omega^{2}\right)-\frac{\Omega^{2} k^{2} \eta}{\rho}=0,
$$

где вводится постоянная, имеющая размерность циклической частоты:

$$
\Omega^{2}=\frac{4 \eta}{\mathfrak{J}} .
$$

Дальнейшее исследование уравнения (19) удобно проводить, вводя следующие обозначения: волновые числа, соответствующие распространению поперечных волн:

$$
k_{\perp}^{\mu \mu}=\frac{\omega}{c_{\perp}^{\mu \mu}}, \quad k_{\perp}^{\mu}=\frac{\omega}{c_{\perp}^{\mu}} ;
$$

отношения скоростей поперечных волн:

$$
{\widetilde{k_{\perp}}}^{2}=\frac{c_{\perp}^{\mu 2}}{c_{\perp}^{\mu \mu 2}}, \quad{\widetilde{k_{\perp}^{\mu}}}^{2}=\frac{c_{\perp}^{2}}{c_{\perp}^{\mu 2}}
$$

характерные безразмерные постоянные:

$$
h_{1}^{2}=\left(\frac{\Omega}{\omega}\right)^{2}, \quad h_{2}^{2}=h_{1}^{2}{\widetilde{k_{\perp}^{\mu}}}^{2}-1-{\widetilde{k_{\perp}}}^{2} .
$$

Тогда в новых переменных уравнение (19) примет более компактный вид:

$$
\frac{k^{4}}{k_{\perp}^{\mu 4}}+h_{2}^{2} \frac{k^{2}}{k_{\perp}^{\mu 2}}+\left(1-h_{1}^{2}\right){\widetilde{k_{\perp}}}^{2}=0 .
$$

Квадраты волновых чисел поперечной плоской гармонической волны находятся из уравнения (20) в виде

где

$$
2 \frac{k^{2}}{k_{\perp}^{\mu 2}}=-h_{2}^{2} \pm \sqrt{\mathfrak{D}}
$$

$$
\mathfrak{D}=h_{2}^{4}-4\left(1-h_{1}^{2}\right){\widetilde{k_{\perp}}}^{2} .
$$

Дискриминант $\mathfrak{D}$ всегда положителен [10], поэтому при произвольной частоте уравнение (20) имеет два вещественных квадрата волновых чисел. С помощью теоремы Виета можно показать, что при $\omega<\Omega$ имеется один положительный квадрат волнового числа $k$, а при $\omega>\Omega-$ два положительных квадрата. Отсюда следует, что при $\omega<\Omega$ существует одна поперечная волна, при $\omega>\Omega-$ две поперечные волны, распространяющиеся без диссипации энергии.

Окончательно можно выписать значения волновых чисел, соответствующих поперечным волнам: если $\omega>\Omega$

если $\omega<\Omega$

$$
\sqrt{2} \frac{k_{1,2 ; 3,4}}{k_{\perp}^{\mu}}= \pm \sqrt{-h_{2}^{2} \pm \sqrt{\mathfrak{D}}}
$$

$$
\left\{\begin{array}{l}
\sqrt{2} \frac{k_{1,2}}{k_{\perp}^{\mu}}= \pm \sqrt{-h_{2}^{2}+\sqrt{\mathfrak{D}}} \\
\sqrt{2} \frac{k_{3,4}}{k_{\perp}^{\mu}}= \pm i \sqrt{h_{2}^{2}+\sqrt{\mathfrak{D}}} .
\end{array}\right.
$$




\section{5. ВОЛНОВЫЕ ЧИСЛА ПЛОСКИХ ГАРМОНИЧЕСКИХ ПРОДОЛЬНЫХ ТЕРМОУПРУГИХ ВОЛН В КОНТИНУУМАХ ПЕРВОГО ТИПА}

Проанализируем второй сомножитель $\operatorname{det} \mathbf{D}_{3}$ в формуле (18). Приравняв нулю определитель матрицы $\mathbf{D}_{3}$, получим бикубическое уравнение для определения волновых чисел плоских гармонических связанных термоупругих волн. Вычислив определитель, имеем:

$$
\begin{gathered}
\operatorname{det} \mathbf{D}_{3}=\left(\rho \omega^{2}-(\lambda+2 \mu) k^{2}\right)\left(\mathfrak{J} \omega^{2}-4 \eta-(\beta+2 \gamma) k^{2}\right)\left(i \frac{\omega}{l_{*}^{2}}-k^{2}\right)- \\
-\frac{i \omega \alpha^{2} k^{2}}{\Lambda_{*}}\left(\mathfrak{J} \omega^{2}-4 \eta-(\beta+2 \gamma) k^{2}\right)-\frac{i \varsigma^{2} k^{2} \omega}{\Lambda_{*}}\left(\rho \omega^{2}-(\lambda+2 \mu) k^{2}\right) .
\end{gathered}
$$

Введем следующие обозначения для упрощения анализа корней уравнения (23): волновые числа:

$$
k_{\|}^{\mu \mu}=\frac{\omega}{c_{\|}^{\mu \mu}}, \quad k_{\|}=\frac{\omega}{c_{\|}}
$$

отношения скоростей поперечных волн:

$$
\widetilde{k_{\|}}=\frac{k_{\|}^{\mu \mu}}{k_{\|}}=\frac{c_{\|}}{c_{\|}^{\mu \mu}}
$$

постоянные, характеризующие связанность механических и тепловых эффектов:

$$
s_{1}^{2}=\frac{\alpha^{2}}{\omega \Lambda_{*} \rho}, \quad s_{1}^{\varsigma 2}=\frac{\varsigma^{2}}{\omega \Lambda_{*} \mathfrak{J}},
$$

безразмерные постоянные:

$$
\begin{gathered}
{\widetilde{k_{1}}}^{2}={\widetilde{k_{\|}}}^{2}\left(1-h_{1}^{2}\right), \quad Q_{1}^{2}=\frac{\omega}{l_{*}^{2} k_{\|}^{2}}, \\
Q_{2}^{2}=Q_{1}^{2}+{\widetilde{k_{\|}}}^{2} s_{1}^{\varsigma 2}+s_{1}^{2}, \quad Q_{3}^{2}=Q_{1}^{2}\left({\widetilde{k_{1}}}^{2}+1\right)+{\widetilde{k_{\|}}}^{2}\left(s_{1}^{\varsigma 2}+s_{1}^{2}\left(1-h_{1}^{2}\right)\right) .
\end{gathered}
$$

В итоге, проведя необходимые аналитические преобразования, получим более компактную форму уравнения $\operatorname{det} \mathbf{D}_{3}=0$ :

$$
\frac{k^{6}}{k_{\|}^{6}}-\left(1+{\widetilde{k_{1}}}^{2}+i Q_{2}^{2}\right) \frac{k^{4}}{k_{\|}^{4}}+\left({\widetilde{k_{1}}}^{2}+i Q_{3}^{2}\right) \frac{k^{2}}{k_{\|}^{2}}-i Q_{1}^{2}{\widetilde{k_{1}}}^{2}=0 .
$$

Заменой

$$
\frac{k^{2}}{k_{\|}^{2}}=Y+\frac{1+{\widetilde{k_{1}}}^{2}+i Q_{2}^{2}}{3}
$$

можно свести бикубическое уравнение (24) к неполному кубическому уравнению:

$$
Y^{3}+p Y+q=0
$$

где коэффициенты уравнения

$$
p=\operatorname{Re} p+i \operatorname{Im} p, \quad q=\operatorname{Re} q+i \operatorname{Im} q
$$

определяются формулами

$$
\begin{gathered}
\operatorname{Re} p={\widetilde{k_{1}}}^{2}-\frac{1}{3}\left(\left(1+{\widetilde{k_{1}}}^{2}\right)^{2}-Q_{2}^{4}\right), \\
\operatorname{Im} p=Q_{3}^{2}-\frac{2}{3} Q_{2}^{2}\left(1+{\widetilde{k_{1}}}^{2}\right),
\end{gathered}
$$




$$
\begin{aligned}
& \operatorname{Re} q=\left(-\frac{2}{27}{\widetilde{k_{1}}}^{4}+\frac{1}{9}{\widetilde{k_{1}}}^{2}+\frac{1}{9}+\frac{2}{9} Q_{2}^{4}\right){\widetilde{k_{1}}}^{2}-\frac{2}{27}-\frac{1}{3} Q_{3}^{2} Q_{2}^{2}+\frac{2}{27} Q_{2}^{4}, \\
& \operatorname{Im} q=\left(\frac{1}{3} Q_{3}^{2}-Q_{1}^{2}-\frac{1}{9} Q_{2}^{2}-\frac{2}{9}{\widetilde{k_{1}}}^{2} Q_{2}^{2}\right){\widetilde{k_{1}}}^{2}-\frac{2}{9} Q_{2}^{2}+\frac{1}{3} Q_{3}^{2}+\frac{2}{27} Q_{2}^{6} .
\end{aligned}
$$

Решение неполного кубического уравнения (25) можно найти согласно формулам Кардано. Приведем указанное решение в канонической алгебраической форме:

$$
Y_{1}=a+b, \quad Y_{2,3}=-\frac{1}{2}(a+b) \pm i \frac{\sqrt{3}}{2}(a-b)
$$

где

$$
\begin{gathered}
a=\sqrt[3]{-\frac{q}{2}+\sqrt{\mathfrak{D}_{1}}}, \quad b=\sqrt[3]{-\frac{q}{2}-\sqrt{\mathfrak{D}_{1}}}, \quad \mathfrak{D}_{1}=\frac{q^{2}}{4}+\frac{p^{3}}{27} \\
\operatorname{Re} \mathfrak{D}_{1}=\frac{1}{4}(\operatorname{Re} q)^{2}-\frac{1}{4}(\operatorname{Im} q)^{2}+\frac{1}{27}(\operatorname{Re} p)^{3}-\frac{1}{9}(\operatorname{Re} p)(\operatorname{Im} p)^{2} \\
\operatorname{Im} \mathfrak{D}_{1}=\frac{1}{2}(\operatorname{Re} q)(\operatorname{Im} q)+\frac{1}{9}(\operatorname{Re} p)^{2}(\operatorname{Im} p)-\frac{1}{27}(\operatorname{Im} p)^{3} .
\end{gathered}
$$

Достаточно выбрать одно из значений квадратного корня $\sqrt{\mathfrak{D}_{1}}$. Воспользуемся далее известной формулой для нахождения квадратного корня из комплекснозначного выражения $p=\operatorname{Re} p+i \operatorname{Im} p$. Положив $\sqrt{p}=z=\operatorname{Re} z+i \operatorname{Im} z$, имеем ровно два значения для $\sqrt{p}$, которые вычисляются согласно формулам

$$
\sqrt{2} \operatorname{Re} z= \pm \sqrt{\operatorname{Re} p+\sqrt{(\operatorname{Re} p)^{2}+(\operatorname{Im} p)^{2}}}, \quad \operatorname{Im} z=\frac{\operatorname{Im} p}{2 \operatorname{Re} z}
$$

Находим также

$$
\begin{aligned}
\sqrt{2} \operatorname{Re} \sqrt{\mathfrak{D}_{1}}= & \sqrt{\operatorname{Re} \mathfrak{D}_{1}+\sqrt{\left(\operatorname{Re} \mathfrak{D}_{1}\right)^{2}+\left(\operatorname{Im} \mathfrak{D}_{1}\right)^{2}}} \\
& \operatorname{Im} \sqrt{\mathfrak{D}_{1}}=\frac{\operatorname{Im} \mathfrak{D}_{1}}{2 \operatorname{Re} \sqrt{\mathfrak{D}_{1}}} .
\end{aligned}
$$

Применяя формулы (26) для каждого из трех значений величины $a$, необходимо подбирать такое значение $b$, для которого выполняется условие

$$
a b=-p / 3
$$

Следуя указанной схеме, получаются все три корня неполного кубического уравнения.

Остается разрешить использованную выше подстановку относительно волнового числа и получить окончательные формулы:

$$
\frac{k_{1,2,3 ; 4,5}}{k_{\|}}= \pm \sqrt{Y_{1,2,3}+\frac{1+{\widetilde{k_{1}}}^{2}+i Q_{2}^{2}}{3}} .
$$

Значения волновых чисел (22), (28), полученные при исследовании бикубического (24) и биквадратного (20) уравнений, можно впоследствии использовать при отделении однозначных ветвей многозначных квадратных и кубических радикалов на комплексной плоскости $k=\operatorname{Re} k+i \operatorname{Im} k$.

Работа выполнена при частичной финансовой поддержке Российского фонда фундаментальных исследований (проект 13-01-00139 «Гиперболические тепловые волны в твердых телах с микроструктурой»).

\section{Библиографический список}

1. Cosserat E. et F. Theorie des corps deformables. Paris : an elastic solid // J. Therm. Stress. 1992. Vol. 15. P. 253Librairie Scientifique A. Hermann et Fils, 1909. 226 p. 264.

2. Green A. E., Naghdi P. M. On undamped heat waves in 3. Green A. E., Naghdi P. M. Thermoelasticity without 
energy dissipation // J. Elasticity. 1993. Vol. 31. P. 189208.

4. Радаев Ю. Н., Семенов Д. А. Гармонические связанные СТЕ-термоупругие волны в свободном цилиндрическом волноводе // Вестн. Самар. гос. ун-та. Естественнонауч. сер. 2008. № 8/1(67). С. 411-459.

5. Ковалев В. А., Радаев Ю. Н., Семенов Д. А. Связанные динамические задачи гиперболической термоупругости // Изв. Сарат. ун-та. Нов. сер. Сер. Математика. Механика. Информатика. 2009. Т. 9, вып. 4, ч. 2. C. $94-128$.

6. Ковалев В. А., Радаев Ю. Н. Волновые числа плоских GNIII-термоупругих волн и неравенства, обеспе- чивающие их нормальность // Изв. Сарат. ун-та. Нов. сер. Сер. Математика. Механика. Информатика. 2010. Т. 10, вып. 3. С. 46-53.

7. Ковалев В. А., Радаев Ю. Н. Волновые задачи теории поля и термомеханика. Саратов : Изд-во Сарат. ун-та, 2010. 328 c.

8. Nowacki $W$. Theory of asymmetric elasticity. Oxford : Pergamon Press, 1986. 384 p.

9. Eringen A. C. Microcontinuum field theories. Vol. 1. Foundations and Solids. Berlin ; Heidelberg ; N. Y. : Springer, 1999. 325 p.

10. Новацикй В. Теория упругости. М. : Мир, 1975. $872 \mathrm{c}$.

\title{
A Mathematical Theory of Plane Harmonic Coupled Thermoelastic Waves in Type-I Micropolar Continua
}

\begin{abstract}
V. A. Kovalev ${ }^{1}$, E. V. Murashkin ${ }^{2}$, Yu. N. Radayev ${ }^{3}$
${ }^{1}$ Moscow Metropolitan Governance University, 28, Sretenka str., 107045, Moscow, Russia, vlad_koval@mail.ru

${ }^{2}$ Institute for Problems in Mechanics of RAS, 101-1, Vernadskogo ave., 119526, Moscow, Russia, murashkin@ipmnet.ru, evmurashkin@gmail.com

${ }^{3}$ Institute for Problems in Mechanics of RAS, 101-1, Vernadskogo ave., 119526, Moscow, Russia, radayev@ipmnet.ru, y.radayev@gmail.com

The present paper is devoted to an analysis of plane harmonic coupled thermoelastic waves of displacements, microrotations and temperature propagating in continua. The analysis is carried out in the framework of linear type-I (GNI/CTE) theory of thermoelastic micropolar continuum. Additional microrotations and moment stresses are taken into consideration. Propagating wave surfaces of weak discontinuities of displacements, microrotations, and temperature are studied by compatibility conditions technique due to Hadamard and Thomas. Wavenumbers (complex propagation constants (CPC)) of plane harmonic coupled thermoelastic waves are obtained. In order to determine the wavenumbers a bicubic and a biquadratic algebraic equations are derived for waves of displacements, microrotations, and temperature. Those equations are then analyzed by the computer algebra system Mathematica. Algebraic forms expressed by complex multivalued square and cubic radicals are obtained for wavenumbers of transverse and longitudinal thermoelastic waves.
\end{abstract}

Key words: micropolar thermoelasticity, type-I continuum, weak discontinuity, longitudinal wave, transverse wave, wavenumber, frequency equation.

\section{References}

1. Cosserat E. et F. Theories of deformable bodies. Paris, Scientific Library A. Hermann et Fils, 1909, 242 p.

2. Green A. E., Naghdi P. M. On undamped heat waves in an elastic solid. J. Therm. Stress. 1992, vol. 15, pp. 253264.

3. Green A. E., Naghdi P. M. Thermoelasticity without energy dissipation. J. Elasticity. 1993, vol. 31, pp. 189208.

4. Radayev Yu. N., Semenov D. A. Harmonic coupled CTE-thermoelastic waves in a free cylindrical waveguide. Vestn. Samar. Gos. Univ. Natural sciences ser., 2008, no. 8/1(67), pp. 411-459 (in Russian).

5. Kovalev V. A., Radayev Yu. N., Semenov D. A. Coupled dynamic problems in hyperbolic thermoelasticity. Izv. Sarat. Univ. (N.S.), Ser. Math. Mech. Inform., 2009, vol. 10, iss. 4, pt. 2, pp. 94-128 (in Russian).

6. Kovalev V. A., Radayev Yu. N. Wavenumbers of plane GNIII-thermoelastic waves and inequality, providing their normality. Izv. Sarat. Univ. (N. S.), Ser. Math. Mech. Inform., 2010, vol. 10, iss. 3, pp. 46-53 (in Russian).

7. Kovalev V. A., Radayev Yu. N. Volnovye zadachi teorii polia $i$ termomekhanika [Wave problems of field theory and thermomechanics]. Saratov, Saratov. Univ. Press, 2010, 328 p. (in Russian).

8. Nowacki W. Theory of asymmetric elasticity. Oxford, Pergamon Press, 1986, 384 p.

9. Eringen A. C. Microcontinuum field theories. Vol. 1. Foundations and Solids. Berlin ; Heidelberg ; N. Y., Springer, 1999, 325 p.

10. Nowacki W. Theory of elasticity. Moscow, Mir, 1975, 872 p. (in Russian). 